\title{
EXTRAVERSION AND THE P300 IN A VISUAL CLASSIFICATION TASK
}

\author{
Georg STEnberg \\ Department of Clinical Neurophysiology, University Hospital, University of Lund, 22185 Lund, Sweden
}

(Received 12 August 1993)

\begin{abstract}
Summary-Amplitude differences between extraverts and introverts in the P300 component of visual event-related potentials were examined in a picture classification task. A set of pictorial stimuli was used with three types of instructions, requiring responses based on colour, semantic category, or a combination of both criteria. Forty university students ( 22 men, 18 women) served as subjects. Based on their scores on the Eysenck Personality Inventory, they were divided into 3 groups of Low, Medium or High Extraversion. The pictures evoked large late positivities, with higher anplitude in the right hemisphere. P300 amplitude increased with increasing degrees of extraversion. The effect of extraversion on amplitude (High $>$ Medium $>$ Low) was larger for target stimuli in posterior derivations. The extraversion effect was mainly attributable to the impulsivity subtrait. Whereas some earlier studies have found higher P300 amplitudes for introverts in tasks inducing habituation or attention decrement, the present tasks differed by using more varied stimuli and requiring more processing. The results are discussed in relation to theories on the biological basis of extraversion.
\end{abstract}

\section{NTRODUCTION}

For almost as long as there have been methods to measure brain activity, there have been proposals to the effect that individual differences in measured brain activity reflect stable, trait-like differences in psychological functioning. Very early in the history of electroencephalography, it was suggested that the salient individual differences in the EEG could be related to personality traits (Lemere, 1936). Investigations into these relationships were initially scarce, in large part due to the lack of general agreement on what traits would best serve as the putative correlates of brain activity. In the last three decades, an emerging literature has increasingly focused on extraversion in that role. There is strong evidence that extraversion is genetically based (Fulker. 1981; Zuckerman, 1991). Most factor-analytic theories of personality agree that extraversion has a place in a narrow circle of fundamental personality dimensions, although there is still disagreement concerning the number and nature of other traits with which it shares that distinction (Costa \& McCrae, 1992; Eysenck, 1992; Zuckerman, 1992). Furthermore, it has been linked to biological concepts by an influential theory (Eysenck, 1957, 1967).

In its early version, Eysenck's theory (1957) made the assumption that extraverts are more prone to inhibition, and introverts more prone to excitation. Brebner and Cooper (1974) later elaborated on this hypothesis by adding a distinction between stimulus analysis and response organization. However, in Eysenck's own theory, the excitation/inhibition formulation was superseded by the arousal theory (Eysenck. 1967), according to which extraversion/introversion is related to cortical arousal. Introverts are assumed to have chronically higher levels of arousal than extraverts, and the behavioural differences between the groups are thought to emanate from this relationship.

Empirical investigations using the EEG to assess the relation between arousal and extraversion have, in the main, been supportive of the hypothesis, but remaining inconsistencies have prompted clarification. After reviewing the literature, Gale (1983) has suggested that variations in task requirements account for the differences in outcome, whereas O'Gorman (1984) has emphasized the importance of the personality measure used. More specifically, recent investigations ( $O^{\prime}$ Gorman \& Lloyd, 1987; Stenberg, 1992) have tended to show that the subtrait impulsivity, which forms part of the broader trait of extraversion, bears a stronger relation to low arousal than extraversion does.

Other means of assessing brain activity have also been applied to the question of the biological basis of extraversion. The overall level of cerebral blood flow (CBF) has been found to correlate with introversion, as predicted by the arousal hypothesis, in a sample of women (Mathew. Weinman \& Barr, 1984), and among women, but not men. in a mixed-sex group (Stenberg. Wendt \& Risberg, 1993). Regional patterns of CBF indicate higher activity in the temporal lobes for introverts than extraverts 
(Stenberg, Risberg, Warkentin \& Rosén, 1990b; Stenberg et al., 1993), an effect which seems pertinent to Gray's $(1972,1981)$ modification of the extraversion/introversion theory.

Event-related potentials (ERPs) have been used to investigate the heightened perceptual sensitivity in introverts which is evident in many behavioural tasks (Siddle, Morrish, White \& Mangan, 1969; Stelmack \& Campbell, 1974). Auditory ERPs have tended to show higher amplitudes for introverts (Bruneau, Roux. Perse \& Lelord, 1984; Stelmack, Achorn \& Michaud, 1977; Stelmack \& Michaud, 1985), as predicted by the arousal theory. The same is true for visual evoked responses over modality-specific visual areas (Soskis \& Shagass, 1974; Stenberg, Rosén \& Risberg, 1988, 1990a). However, these effects have been importantly modified by psychological factors such as attention and habituation (Stelmack \& Michaud, 1985; Stenberg et al., 1990a). For this reason, investigations into the later, more clearly endogenous, components of the evoked response seem to be of interest.

The P300 is the most extensively investigated of the psychologically important ERP components (Picton \& Hillyard, 1988). It is known to accompany cognitive processing of stimuli, and its size is eminently sensitive to factors such as subjective expectancy. Its functional significance remains, however, in dispute. An influential hypothesis proposes that the P300 arises when there is a need to update the internal model of the environment in working memory (Donchin \& Coles, 1988), whereas an alternative proposal, the context closure hypothesis (Verleger, 1988), regards the P300 as the sign of a closed perceptual epoch, i.e. a reflection of confirmed, rather than revised, subjective expectancies. Irrespective of its functional description in psychological terms, the physiological process underlying the P300 seems to be best described as a momentary deactivation of wide-spread cortical areas (Birbaumer. Elbert, Canavan \& Rockstroh, 1990; Verleger, 1988). The reduction of excitability is thought to serve the selective enhancement of relevant processing, accomplished by a generalized inhibition of other, potentially competing, cell assemblies (Birbaumer \& Elbert, 1988: Birbaumer et al., 1990).

In view of its psychological significance, and its relevance to the question of excitation/inhibition propensities, it seems that the P300 component is an appropriate object of study for investigations into the physiology of extraversion, and a number of studies on this topic have appeared during recent years (Cahill \& Polich, 1992; Daruna, Karrer \& Rosen, 1985; Ditraglia \& Polich, 1991; O'Connor, 1983: Orlebeke, Kok \& Zeillemaker, 1989: Polich \& Martin, 1992; Pritchard, 1989: Wilson \& Languis, 1990). The studies have generally adhered to the auditory oddball paradigm, or some variation thereof. In this paradigm, the $S$ listens, with closed eyes, to a sequence of tone occurrences, in which one tone is represented frequently, and another one rarely. The P300 is recorded in response to the rare tone, which is usually the target of some task, such as counting or button-pressing. The rationale for expecting differences between extraverts and introverts to emerge in this task is based on observations of extraverts having a faster habituation rate (O'Gorman, 1977) and a more rapid performance decline in vigilance tasks demanding sustained attention (Keister \& McLaughlin, 1972). Since the typical auditory oddball task tends to induce monotony, introverts are expected to sustain their attention better, leading to higher amplitudes in the averaged ERP. Daruna et al. (1985) found the expected result in a stimulus-guessing task, as did Wilson and Languis (1990) in a conventional oddball task. However, in the same paradigm, Polich and Martin (1992) obtained the expected negative correlation between extraversion and P300 amplitude only for males, and in Pritchard's study (1989) there was no correlation between amplitude and extraversion. Subject selection procedures, such as extreme groups versus median-split of unselected groups, may be an explanatory factor in accounting for varying outcomes.

The importance of time-on-task was explicitly studied by Ditraglia and Polich (1991), who used two trial blocks, and found declining P300 amplitudes over time for extraverts, but not for introverts, consistent with their hypothesis of accelerated habituation in extraverts being the determining factor.

Cahill and Polich (1992) manipulated the factor of stimulus probability, while keeping time-on-task to a minimum to avoid habituation effects. Under these circumstances, extraverts tended to have higher P300 amplitudes, and they had a significantly stronger probability effect, i.e. a steeper increase of amplitude with diminishing target stimulus probabilities. The results found by Orlebeke et al. (1989) are, in a broad sense, in agreement with those of Cahill and Polich, since they observed stronger differentiation between their signal and non-signal conditions (response required for every stimulus vs no response required) in their High Disinhibition group. Disinhibition is a subscale of the Sensation 
Seeking Scale, for which moderately high correlations with extraversion are normally obtained (Zuckerman, 1991).

Thus, it seems that if recording conditions are monotonous and lead to attrition of attentional resources, extraverts tend to produce smaller P300s than introverts, in keeping with their more rapid decline of signal detection performance (Keister \& McLaughlin, 1972) and electrodermal responses (O'Gorman, 1977) under similar circumstances. If, however, habituation is avoided, or response requirements are kept high, the P300 differences between introverts and extraverts seem to be abolished or reversed. There are some indications that, under these conditions, extraverts may be more sensitive to the task contingencies that produce the P300.

The ubiquitous use of the oddball paradigm may have confounded the question of differences in attention attrition with that of P300 differences between extraverts and introverts per se. The present study attempts to unconfound these issues by using a different procedure to evoke the P300. The use of a more varied set of stimuli and a more cognitively demanding task presumably avoids the gradual decay of attention and performance which accounts for previously observed differences between extraverts and introverts. In so doing, it can address the question of possible personality-related differences in the processes leading to different endogenous ERP components, such as those indicative of attentive processing and those indicative of transient inhibition.

A visual classification task is used here in three varieties, all based on the same set of pictorial stimuli. The pictures are to be classified on the basis of colour (black/white), semantic category (animals/non-animals), or a combination of both criteria. Attentional requirements are kept high, and performance is measured both as regards speed and accuracy. Since attentional resources have often been adduced as an explanation of individual differences in P300 amplitude, it may be important to compare ERP measures with behavioural data. Furthermore, the use of three levels of difficulty will permit the study of possible relations between task difficulty and individual differences in ERPs. Targets and non-targets are made equiprobable, which has the effect of keeping the length of each recording session to the minimum demanded by a satisfactory signal-to-noise ratio.

\section{METHOD}

\section{Subjects}

Forty-one $S$ s volunteered to participate in experiments on brain-waves and mental activity. They were paid for their participation. One $S$ was excluded because of technical malfunctions during the recording session. Of the remaining 40 ss. 22 were male. Mean age was 24.7 years (SD: 5.7, range: $18-46)$.

All $S$ s but one were university students. Given the fact that students following different degree courses have shown consistent personality differences in a number of studies (Wilson, 1981), an effort was made to avoid bias in the personality composition of the sample by including students from different courses. Thus, 13 studied computer science, 10 psychology, 6 neurobiology. and 10 other subjects, mostly medicine. The group averages for EPI extraversion (13.2 \pm 4.0$)$ and neuroticism (7.2 \pm 3.3 ) do not deviate substantially from those of the Swedish standardization group (12.3 \pm 3.8 and $7.6 \pm 4.3$, respectively).

No score on the Lie scale exceeded 6; average was 2.3. In addition to the conventional Extraversion score, separate scores for the subcomponents Impulsivity and Sociability were derived according to Revelle. Humphreys. Simon and Gilliland (1980).

\section{Personality groups}

On the basis of their scores on the EPI Extraversion scale, the $S$ s were divided into a Low (3-11, $14 \mathrm{Ss}$ ), a Medium (12-14, $11 \mathrm{Ss}$ ), and a High (15-21, $15 \mathrm{Ss}$ ) group for Extraversion. (Because of tied ranks, this was the closest approximation to equal group sizes attainable.) A similar tri-partite division was made for the Neuroticism scale (13-13-14 Ss, respectively).

\section{Procedure}

Stimulus material was selected from the set of pictures published by Snodgrass and Vanderwart (1980). This is a set of 260 black-and-white line drawings, representing animals, tools, furniture and 
other everyday objects. From a digitized version of the picture set, a pool of 60 pictures was selected. Of these, 28 represented animals.

The pictures were presented on the screen of an Macintosh Plus computer. They were displayed under program control on the centre of the screen, against a grey background. Each picture was displayed for $1.5 \mathrm{sec}$, followed by $0.5 \mathrm{sec}$ of an empty, grey screen. For each upcoming stimulus presentation, the display program determined, in a quasi-random fashion, whether the picture was to be displayed in black or in white against the grey background. It also determined whether the stimulus was to be selected from a list of animal pictures or from a list of other objects.

The $S$ was instructed to press the mouse button as soon as a picture in the designated target category was shown. The targets were, in the Colour task, all pictures shown in white; in the Semantic task, all pictures representing animals, and in the Colour + Semantic task, all pictures shown in white and representing non-animals. The instructions emphasized accuracy and speed to an equal degree. The three tasks were presented to the $S \mathrm{~s}$ in counterbalanced order.

The proportion of targets was set to 0.5 in all tasks, and the independent probabilities of display mode (black/white) and picture category (animals/non-animals) were adjusted accordingly, i.e. to 0.5 for each in the Colour and the Semantic tasks, and to 0.71 for white display and non-animals in the combined Colour + Semantic task, producing the probability 0.5 for the conjunction. Stimulus presentation proceeded by randomized selection of stimuli according to the set probabilities, until a sufficient number of acceptable ERP sweeps had been collected, with recycling of previously shown stimuli when the stimulus list had been exhausted.

During the experiment, the $S$ sat in a darkened laboratory in a slightly reclining armchair in front of the Macintosh screen, with the right index finger resting on the mouse button. The personality questionnaire EPI, form A, had been completed at home before the laboratory session. All recordings were made in the afternoon.

\section{Data acquisition}

The EEG was recorded, using a BioLogic Brain Atlas III + system, with electrodes applied to all 19 positions of the 10/20-system, except Fpl and Fp2. Electrodes were applied above and beside the right eye to monitor blinks and eye movements. All electrodes were referred to linked earlobes, and a ground electrode was applied to the forehead. Impedances were kept below $6 \mathrm{k} \Omega$. The EEG signals were filtered with a bandpass of $0.1-70 \mathrm{~Hz}(3 \mathrm{~dB}$ attenuation; roll-off $12 \mathrm{~dB} /$ octave $)$ and digitized with a sampling frequency of $250 \mathrm{H} z$ during a period extending from $150 \mathrm{msec}$ pre-stimulus to 870 msec post-stimulus. Data acquisition was triggered from the Macintosh computer by the serial transmission of a byte, which also marked the imminent stimulus as a target or a non-target presentation. Averages were formed on-line and subsequently saved to disk.

\section{Artifact control}

At the start of the measurement session, $S$ s were instructed to blink voluntarily and repeatedly during a 4 sec epoch, which was recorded. This recording was used in two ways. First, the size of the EOG artifacts was used to set the artifact rejection level for the individual $S$. The Brain Atlas uses an algorithm for automatic artifact rejection, which rejects any sweep with a deflection exceeding $98 \%$ of $\mathrm{A} / \mathrm{D}$-converter saturation. Amplifier gain for the EOG channels can therefore be used to adjust the level at which artifacts will trigger sweep rejection. This was done individually, based on the size of the recorded artifacts. The procedure did not, however, lead to complete elimination of artifacts from the averages. In some cases, the settings proved to be too liberal, allowing subthreshold artifacts into the average. In other cases, the procedure was too conservative, resulting in the rejection of a large proportion of sweeps, and automatic artifact rejection had to be disabled at some point to allow completion of the recording within a reasonable time limit.

The recorded voluntary blinking epochs were therefore used to correct the averaged ERPs for remaining artifacts. For each $S$, a regression coefficient was computed for each EEG channel, indicating the extent to which its amplitude could be predicted from the EOG. |Only the vertical EOG channel was used for this purpose, since the horizontal EOG electrode had been misplaced on a few $S$ s and computation of transmission coefficients from the derived bipolar channel VEOG-HEOG (Connolly \& Kleinman, 1978) showed slighty higher variability over individuals than those from the 
VEOG alone]. These regression coefficients. a measure of the transmission of EOG activity to the different electrode positions, showed small variation across individuals, and the expected decline with increasing distance from the eyes. They averaged 0.36 for frontal, 0.13 for central, 0.07 for parietal, and 0.05 for occipital electrode positions. For each averaged ERP, recorded EOG activity was subtracted from each EEG channel after multiplication with the appropriate coefficient. The average of the pre-stimulus epoch was then subtracted from each subsequent data point.

\section{Behavioural data}

$S$ responses were recorded by the Macintosh computer and saved to files. The data included median reaction time across all correct target detections in the task, and the distribution of true/false positive/negative responses. From these data, the $d^{\prime}$ measure of detection sensitivity was computed for each $S$ in each task. In the computations, a correction term of 0.005 was added to the proportion of false alarms, and subtracted from the proportion of hits, to avoid computations involving probabilities of 0.0 or 1.0 , which give undefined results.

\section{Data analysis}

Due to the risk of obtaining falsely significant results in repeated measures ANOVAs if the model assumptions are violated, all probabilities reported here have been adjusted by the Greenhouse-Geisser correction (Vasey \& Thayer, 1987). (Degrees of freedom are, however, reported unadjusted.)

\section{RES ULTS}

\section{Demographic and personality variables}

The sex distribution was not significantly different across Extraversion groups: Low: 10/4 (males/females), Medium: 5/6, High: 7/8; $\chi^{2}(2)=2.35 ; P=0.31$. There were, however, age differences. Mean age for the different groups were: Low: 27.9, Medium: 23.8, High: 22.4 years $[F(2.37)=4.08 ; P=0.03]$. Since age has been found to have an influence on P300 characteristics (Pfefferbaum, Ford, Wenegrat, Roth \& Kopell, 1984), this could, in principle, pose a risk for confounding the effects of age and extraversion, although it is less likely that age-related P300 changes should be a factor of importance within the very narrow age span of a few years in young adulthood which separated the groups in this study. Still, in what follows, age will be introduced as a covariate, where appropriate, as a guard against confounding age and extraversion effects.

The other main personality variable of the EPI questionnaire, Neuroticism, was not correlated with Extraversion in the present sample $(r=-0.18)$, and there were no significant differences in Neuroticism between Extraversion groups: $F(2,37)=1.27$, NS.

\section{Behavioural results}

The reaction time data were analyzed in a two-way ANOVA, using Task and Extraversion as factors. There was no effect of Extraversion $[F(2,37)<1$, NS $]$, but the effect of Task was highly significant $[F(2,74)=182.4, P<0.001]$. The task effect was in the predicted direction, with the more difficult tasks producing longer reaction times (mean RTs were: Colour: 275, Semantic: 397 , and Colour + Semantic: $467 \mathrm{msec}$ ). The interaction of Extraversion and Task was not significant $[F(4,74)=1.47 ; \mathrm{NS}]$. The addition of age as a predictor indicated a reliable increase of reaction times with age $[F(1,36)=7.88, P<0.01]$.

The $\mathrm{d}^{\prime}$ data for accuracy showed no effect of Extraversion $[F(2,37)=1.20, \mathrm{NS}]$, but the predictable effect of decreasing detection sensitivity with increasing task difficulty $[F(2,74)=8.93, P<0.001]$. The interaction of Task and Extraversion was not significant $[F(4,74)<1]$. Neuroticism was, however, related to detection sensitivity, higher degrees of Neuroticism being associated with worse performance $[F(2,37)=3.46, P=0.04]$.

The other signal detector parameter, $\beta$, which indicates bias in the criterion set by the $S$, also changed between tasks $[F(2,74)=4.61, P=0.01]$. With increasing difficulty, $S$ s evidenced increasingly liberal bias, i.e. a lower threshold for calling yes: mean of $\ln (\beta)$ was $-0.24,-0.41$ and -0.69 for the three tasks. There was no relation to personality. 
Although signal detection parameters differed between tasks, the general performance level was high overall, with mean guess-corrected accuracy (hits - false alarms) varying from $98.7 \%$ for Colour, over $97.7 \%$ for Semantic to $96.7 \%$ in Colour + Semantic.

In summary, the manipulation of task difficulty was effective in producing the desired effects on RTs and sensitivity. Neuroticism was related to poor performance, but the extraversion groups did not differ in any aspect of accuracy or reaction speed.

\section{ERP results}

Averaged and artifact-corrected ERP curves for 12 electrode positions are presented in Figs I and 2. In Figure 1, data are averaged over all subjects to show the effects of the task manipulation. In Fig. 2, data are collapsed across tasks, and the three Extraversion groups are presented separately.

The figures show a large late positive complex, which exhibits latency and wave-shape differences between tasks. Amplitudes of this complex are higher for the High Extraversion group thatn for the Medium, and higher for the Medium than for the Low Extraversion group. Electrode site differences include the expected anterior-posterior gradient for the late positivity, as well as a hemispheric difference, with higher amplitudes on the right side.

The analysis will give an initial overview of effects on amplitude and latency for the parietal midline $(\mathrm{Pz})$ electrode, and then proceed with a more fine-grained analysis of time-interval mean amplitude for 8 laterally placed electrodes, allowing assessment of hemispheric as well as anterior-posterior differences.

P300: peak amplitude and latency. Amplitude and latency were determined for the point of maximum positivity in the $200-800 \mathrm{msec}$ range of $\mathrm{Pz}$ channel. The data were analyzed by 3 -way ANOVAs, using Extraversion (3 levels) as a between $S$ s factor, and Task ( 3 levels) and target/Non-target ( 2 levels) as within- $S$ factors.

As suggested by the cell means shown in Table 1, the peak amplitudes showed a significant main effects of Task [decreasing amplitude with increasing task difficulty; $F(2,74)=3.64 ; P=0.039$ ], and Target [larger amplitudes for targets than non-targets, $F(1,37)=27.89 ; P<0.001]$. The interaction was also significant [the easier the task, the larger the target/non-target difference, $F(2.74)=7.29 ; P=0.0011$.

There was a main effect of Extraversion on amplitude $[F(2,37)=4.79 ; P=0.014]$, which is illustrated in Fig. 3. There was also a non-significant trend toward an interaction, Extraversion $\times$ Target $[F(2,37)=2.63 ; P=0.086]$.

The latency data showed the expected main effect of Task [increasing latency with increasing task difficulty. means: 392,482 and $502 \mathrm{msec}, F(2,74)=33.95 ; P<0.001]$. No effect involving Extraversion was significant.

Time interval mean amplitudes. Mean amplitudes were computed for two intervals: $300-500$ and 500-700 msec. This was done for 8 electrodes: F3, F4, C3, C4, P3, P4. O1 and O2, to allow study of differences between hemispheres as well as gradients along the anterior-posterior axis. Thus, for each time interval, a 5-way ANOVA was computed, using Extraversion ( 3 levels), Task ( 3 levels), Target/Non-target ( 2 levels), Hemisphere (2 levels) and Anterior/Posterior location (4 levels) as factors. Results involving within $S$ effects are given in Table 2 . Since these effects are not the primary concern in the present study, only a brief description of the main effects will be given in the following paragraph.

The Hemisphere effects were due to larger amplitudes over the right hemisphere in both time intervals. Amplitudes were larger for targets than for non-targets. The task effects were different in the two intervals. Amplitudes decreased with increasing task difficulty in the $300-500$ interval. and increased in the 500-700 band. Amplitudes increased posteriorly in both bands, reaching an occipital maximum in the earlier interval. but a parietal maximum in the later one.

The results concerning Extraversion are given in Table 3. The significant main effects for both intervals are illustrated in Fig. 3 ( $b$ and $c$ ), which show increasing amplitudes with increasing degrees of Extraversion. The pattern of significant interaction effects indicates that the differences between the personality groups are related to the classical P300 (P3b). Two distinguishing marks for the P3b are its anterior--posterior gradient and the target effect operating on its amplitude (targets $>$ nontargets). Both of these characteristics are more accentuated in the more extraverted Ss. These aspects 
Task effects : target stimuli
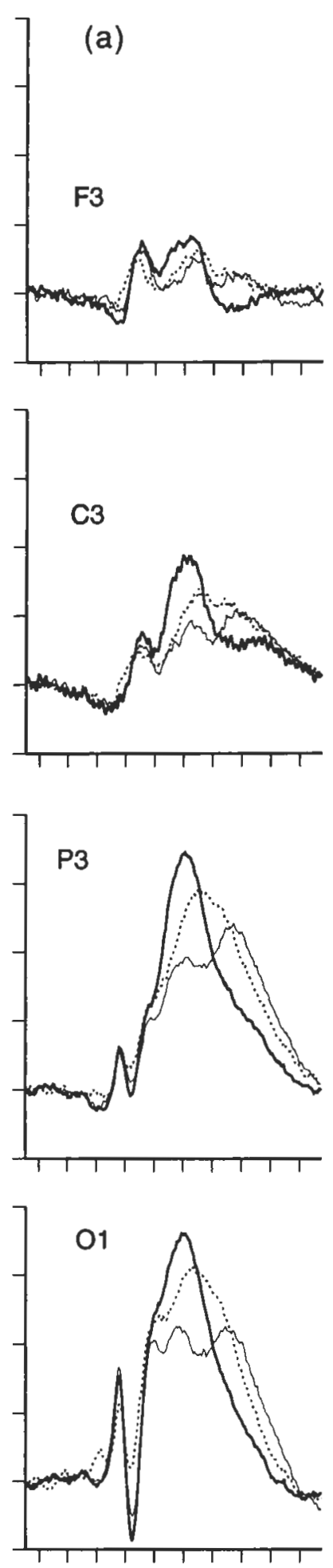

Fz
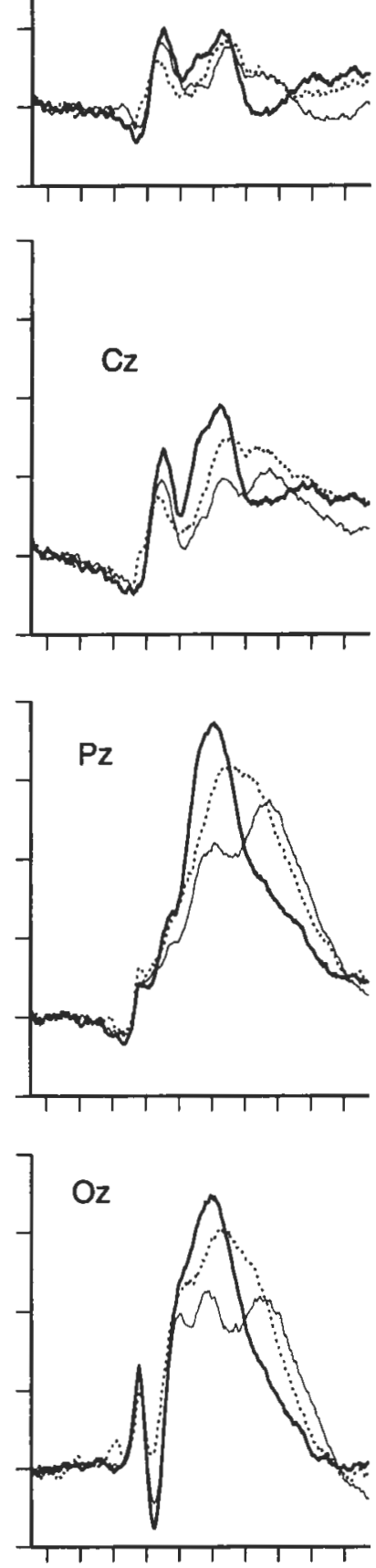
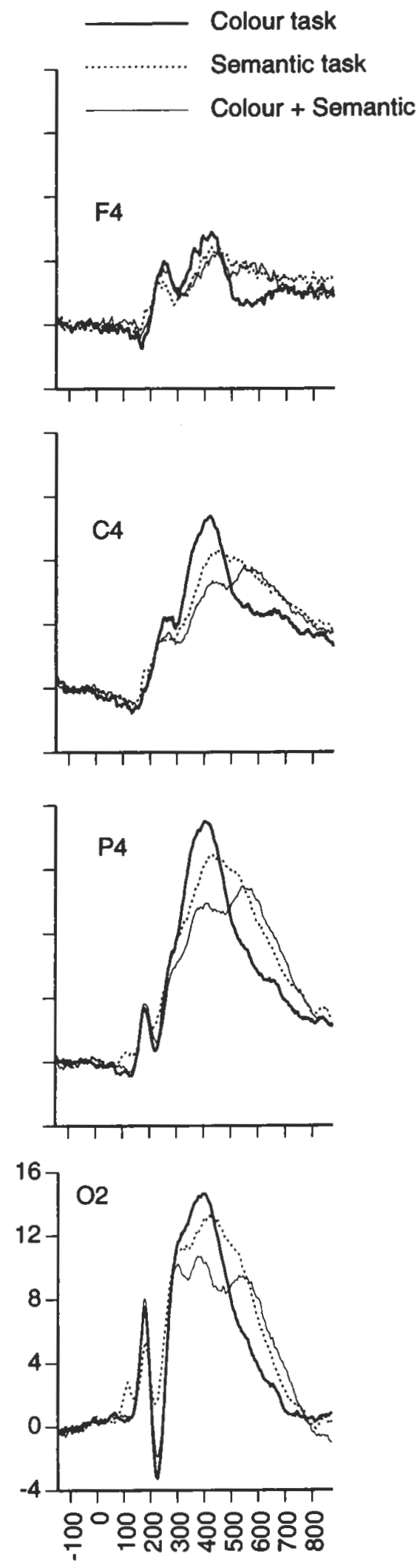

Fig. I (a)-legend overleaf. 
Task effects : non-target stimuli

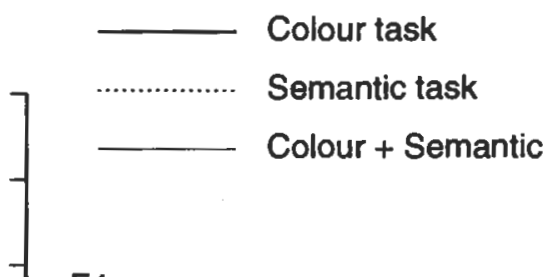

F3

(b)

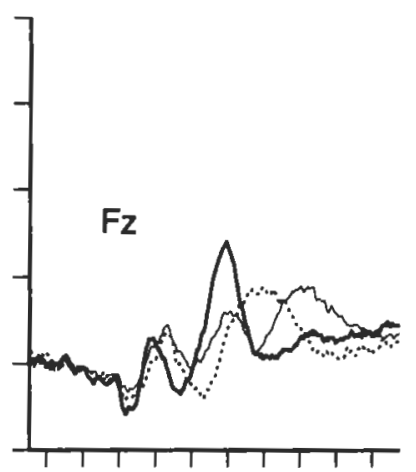

F4
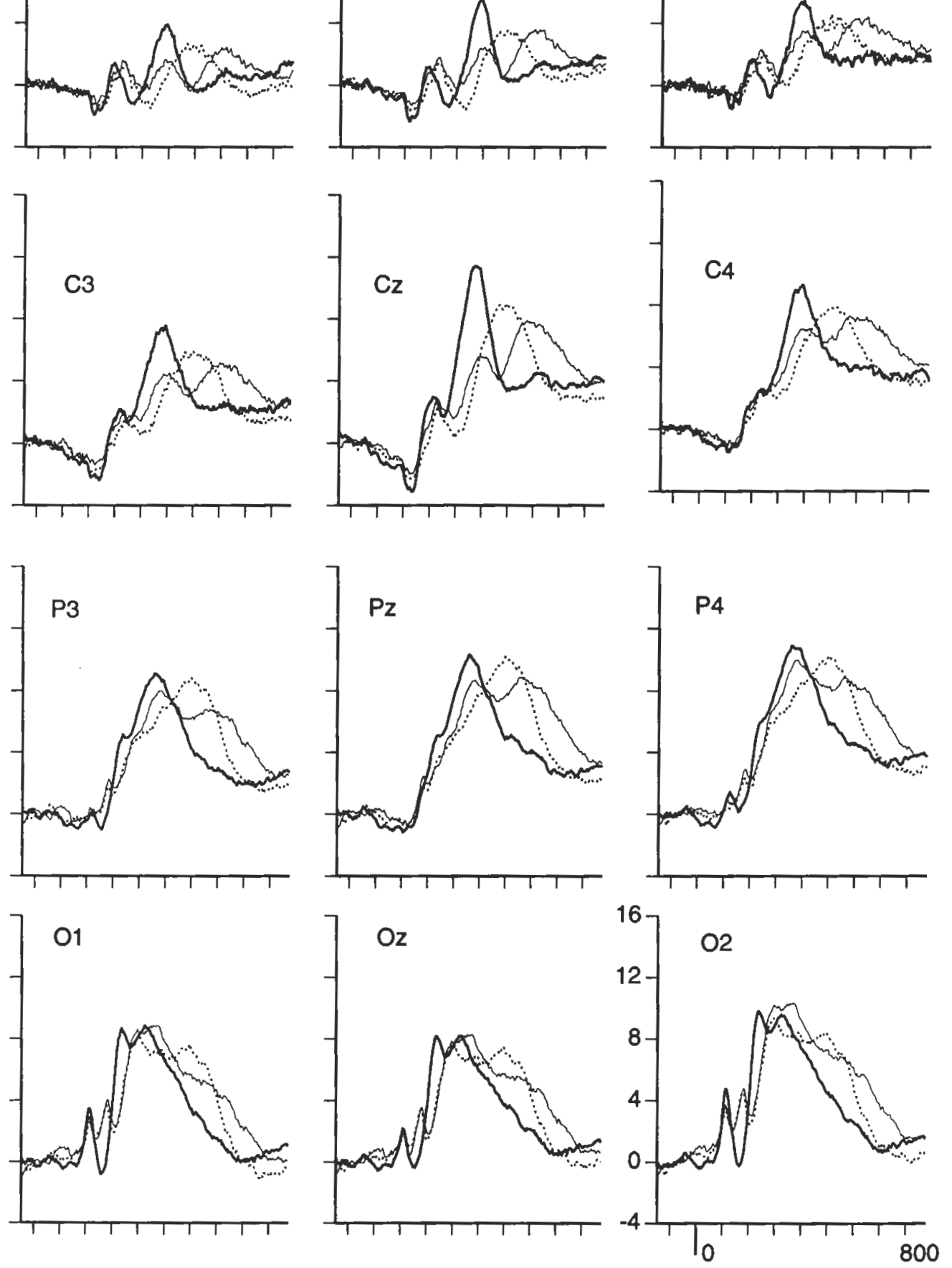

Fig. I(b)

Fig. I. ERP wave forms averaged over all $S$ s for the three conditions. (a) Target stimuli, (b) non-targets. 
Extraversion effects : target stimuli
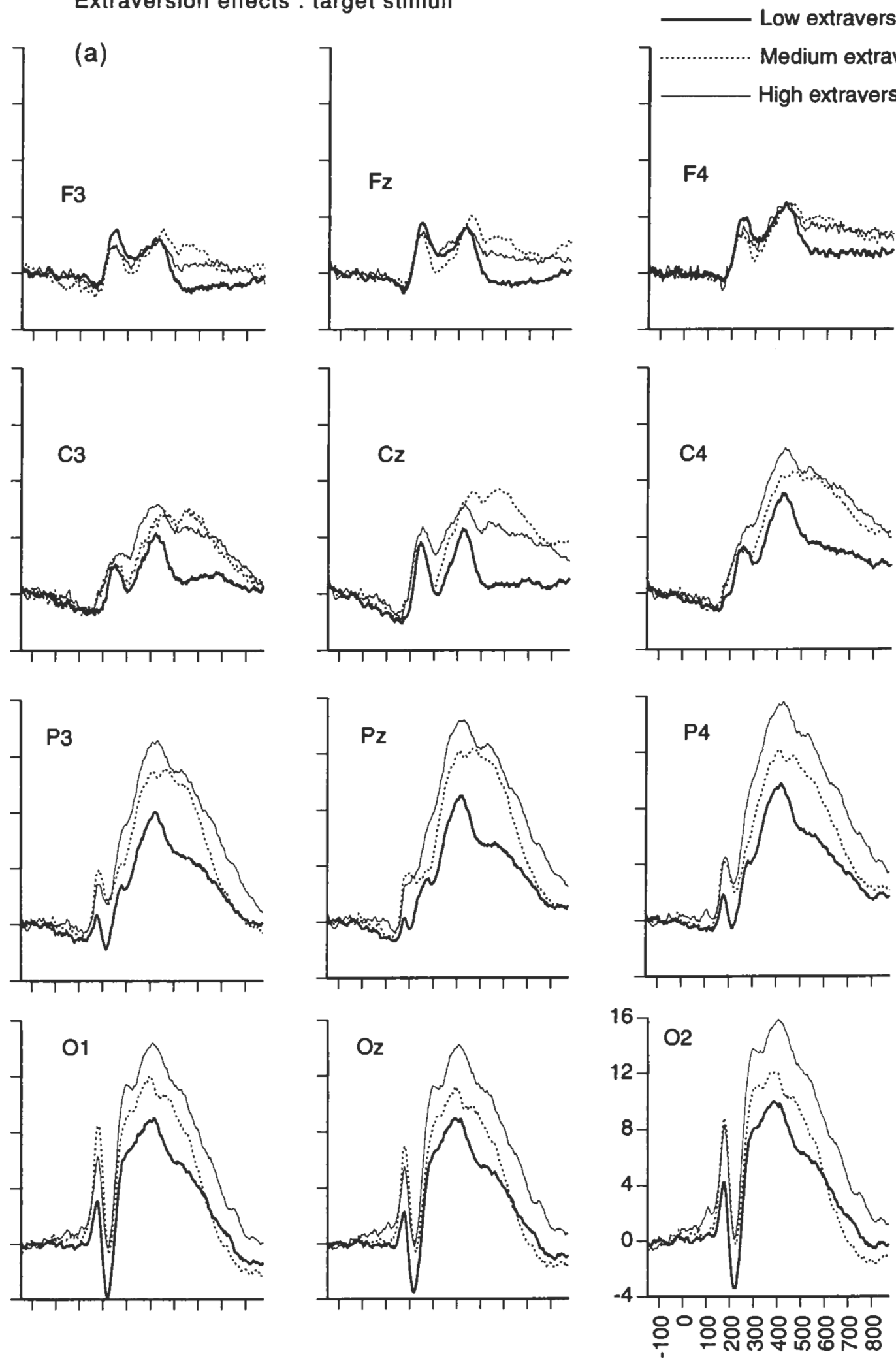

Fig. 2(a)-legend overleaf. 
Extraversion effects : non-target stimuli
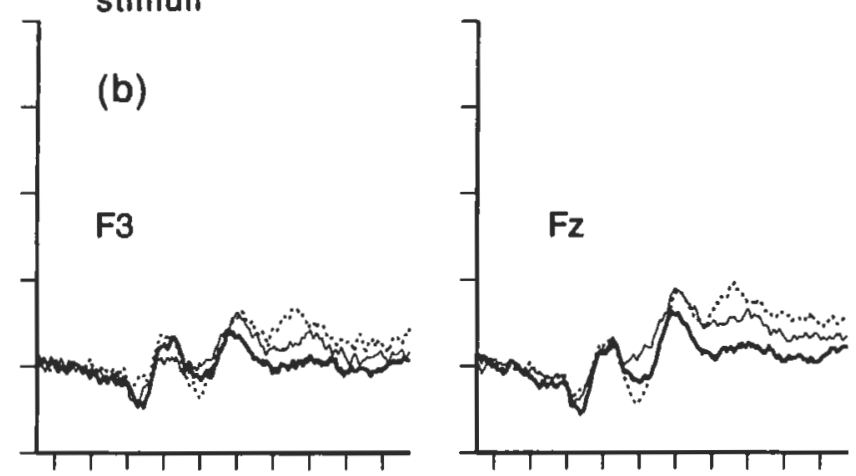

Low extraversion

Medium extraversion
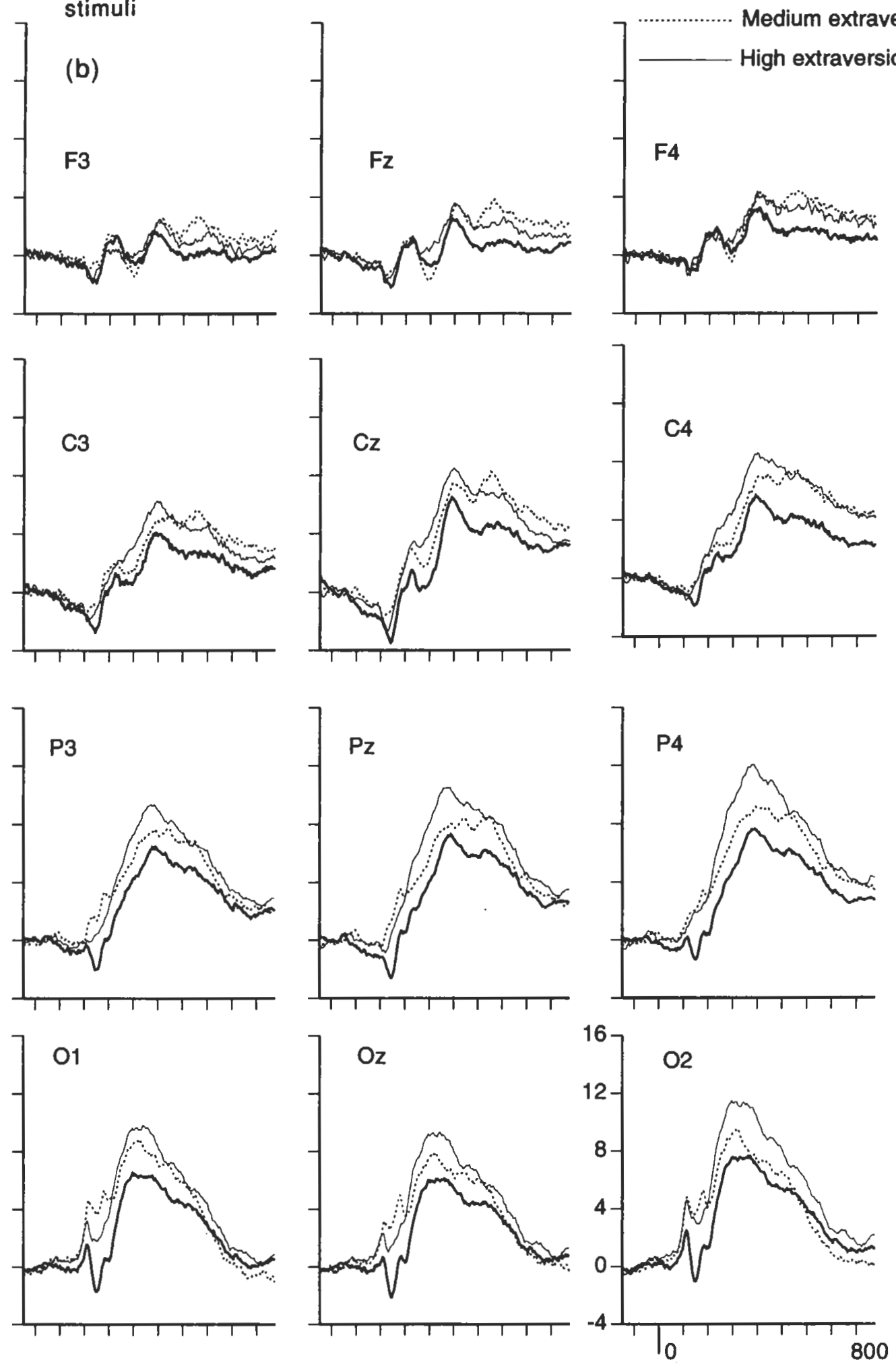

Fig. 2(b)

Fig. 2. ERP wave forms for the three Extraversion groups, averaged over tasks. (a) Target stimuli, (b) non-targets. 
Table 1. Means and standard errors of peak P300 amplitude at Pz for the three Extraversion groups

\begin{tabular}{|c|c|c|c|c|c|c|}
\hline \multirow[b]{2}{*}{ Extraversion } & \multicolumn{2}{|c|}{ Colour } & \multicolumn{2}{|c|}{ Semantic } & \multicolumn{2}{|c|}{ Colour + semantic } \\
\hline & Target & No target & Target & No target & Target & No target \\
\hline Low & $13.85(1.21)$ & $11.87(1.43)$ & $11.84(1.45)$ & $10.88(1.19)$ & $10.34(1.17)$ & $9.50(0.60)$ \\
\hline Medium & $17.65(2.58)$ & $11.61(1.92)$ & $16.89(2.23)$ & $12.70(1.34)$ & $15.46(2.25)$ & $12.79(1.38)$ \\
\hline High & $19.93(1.71)$ & $14.28(1.53)$ & $18.32(1.32)$ & $14.46(1.09)$ & $17.06(1.32)$ & $14.63(0.79)$ \\
\hline
\end{tabular}
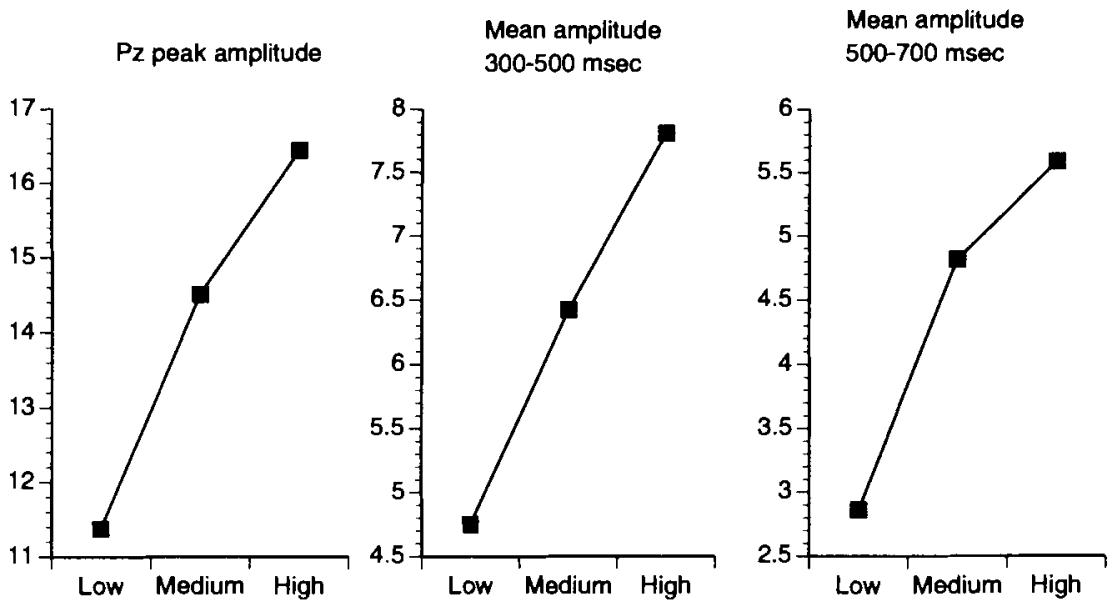

Fig. 3. Average amplitudes for 3 Extraversion groups: left: peak amplitude for the Pz electrode; center: mean amplitude in 300-500 msec interval, averaged over 8 recorded electrodes; right: mean amplitude in 500-700 msec interval, averaged over 8 recorded electrodes.

of the data are illustrated in Fig. 4. As demonstrated by the significant Extraversion $\times$ Target $\times$ Anterior/Posterior interaction, the differences between Extraversion groups are largest for target stimuli in posterior derivations. For both time bands, there is also a significant interaction between Extraversion and Anterior/Posterior location (greater group differences posteriorly), and for the later time band an Extraversion $\times$ Target/Non-Target effect as well (greater group differences for target stimuli). These simpler interactions may suggest that the target and gradient effects to some extent separate the Extraversion groups independently of each other, but the lower-order interactions, as well as the significant main effects, should be interpreted with caution in the presence of the significant 3-way interaction.

Table 2. Within $S$ effects on ANOVAs for time interval mean amplitudes in the 300-500 and 500-700 msec bands

\begin{tabular}{|c|c|c|c|c|c|}
\hline & \multirow[b]{2}{*}{$d f$} & \multicolumn{2}{|c|}{$300-500$} & \multicolumn{2}{|c|}{$500-700$} \\
\hline & & $r$ & $P(\mathrm{G}-\mathrm{G})$ & $F$ & $P(\mathrm{G}-\mathrm{G})$ \\
\hline Anterior-Posterior location & 3,111 & 107.95 & $<0.001$ & 53.09 & $<0.00 \mathrm{l}$ \\
\hline Task & 2.74 & 11.93 & $<0.00 \mid$ & 30.27 & $<0.001$ \\
\hline Target/Non-larget & 1,37 & 23.67 & $<0.001$ & 9.51 & 0.004 \\
\hline Hemisphere & 1,37 & 98.20 & $<0.001$ & 102.09 & $<0.001$ \\
\hline Ant/Post $\times$ Task & 6,222 & 8.47 & $<0.001$ & 6.54 & 0.001 \\
\hline AndPost $\times$ Turget & 3.111 & 56.86 & $<0.001$ & 30.46 & $<0.001$ \\
\hline Talsk $\times$ Target & 2.74 & 21.08 & $<0.001$ & 0.62 & - \\
\hline AndPost $\times$ Hemi & 3.111 & 6.69 & 0.004 & 11.26 & $<0.001$ \\
\hline Task $\times$ Hemi & 2.74 & 3.45 & 0.041 & 2.17 & - \\
\hline Target $\times \mathrm{Hemi}$ & 1,37 & 5.19 & 0.029 & 0.06 & - \\
\hline An/Post $\times$ Task $\times$ Target & 6,222 & 26.26 & $<0.001$ & 1.17 & - \\
\hline AnuPost $\times$ Targel $\times$ Hemi & 3,111 & 13.17 & $<0.001$ & 19.47 & $<0.00 \mid$ \\
\hline
\end{tabular}

Only row: showing significant elfects have been included. $P(\mathrm{G}-\mathrm{G})$ indicutes probability after Greenhouse-Geisser correction 

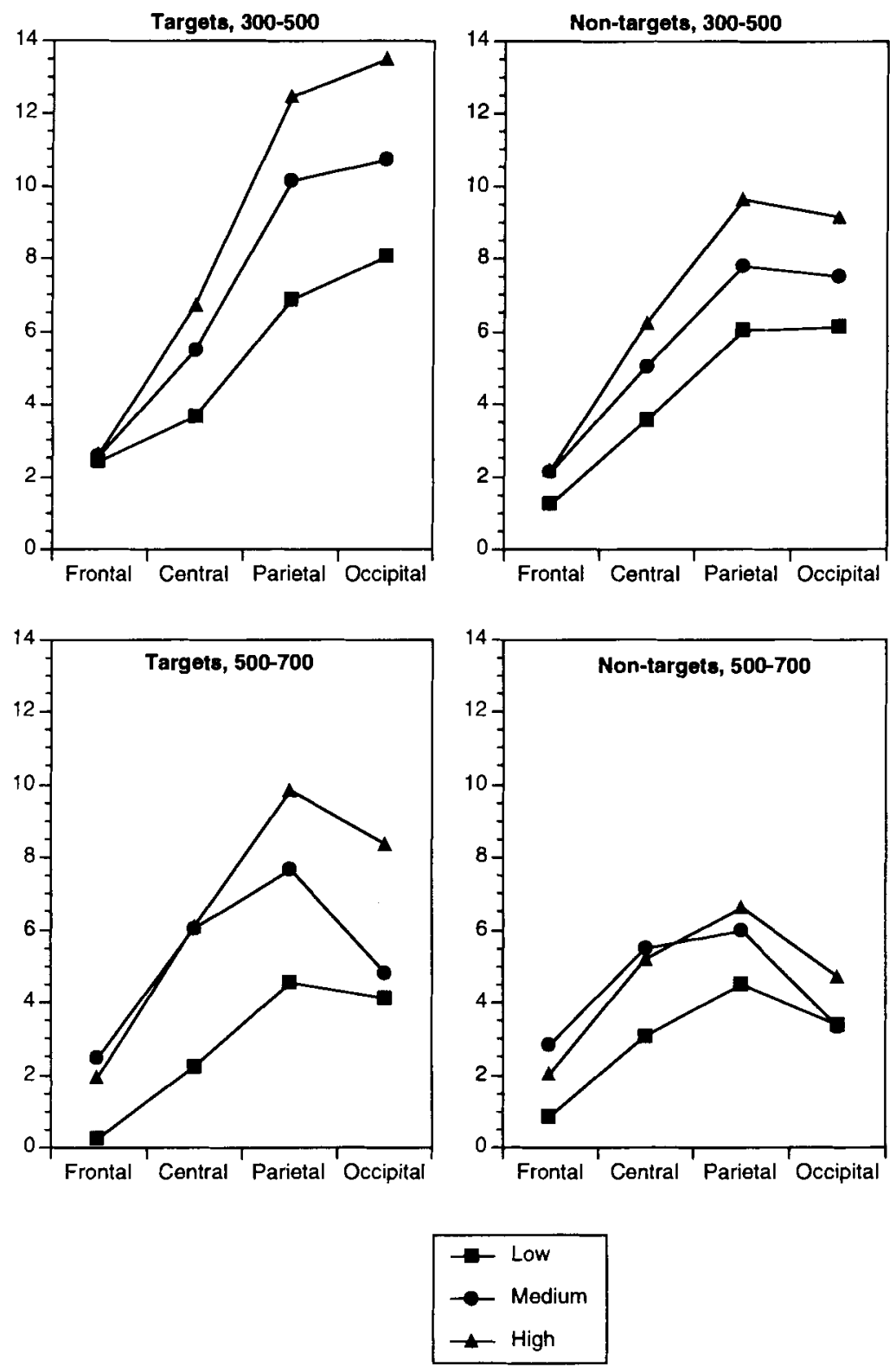

Fig. 4. Illustration of the Extraversion $\times$ Target/Non-target $\times$ Anterior/Posterior interaction Average amplitude in time bands for Low, Medium and High Extraversion groups.

Extraneous variables. In order to assess the influence of some potentially confounding variables, a few additional analyses were made. Thus, as described in the Methods section, stimulus presentation had continued until an acceptable number of unrejected sweeps had been assembled. Due to the varying incidence of artifacts, this meant that the number of presented stimuli was not fixed. Stimulus repetitions could, in principle, affect amplitude, because it has been shown that the late positivity is enhanced for repeated stimuli. Therefore, the number of presented stimuli was examined for each $S$ and each task. However, no differences were found, either between tasks or between Extraversion groups, in the number of stimuli delivered. The average number of presented stimuli were: Colour task: $169 \pm 55$ (mean \pm SD), Semantic: $167 \pm 58$, and Colour + Semantic: $169 \pm 44$. In a Task $\times$ Extraversion ANOVA, all $F$ s were $<1$ and non-significant.

Furthermore, concerns could be raised about the possibility of differing blink rates, and consequent contamination of ERP amplitudes with artifacts. However, as can be seen in Figs 1 and 2, the correction procedure was successful in eliminating the influence of EOG artifacts. (If such artifacts are present. 
Table 3. Between $S$ effects in ANOVAs for time interval mean amplitudes in the $300-500($ and $500-700 \mathrm{msec}$ bands

\begin{tabular}{|c|c|c|c|c|c|}
\hline & \multirow[b]{2}{*}{$d f$} & \multicolumn{2}{|c|}{$300-500$} & \multicolumn{2}{|c|}{$500-700$} \\
\hline & & $F$ & $P(\mathrm{G}-\mathrm{G})$ & $F$ & $P(\mathrm{G}, \mathrm{G})$ \\
\hline Extraversion & 2.37 & 5.50 & 0.008 & 4.96 & 0.012 \\
\hline Extraversion $\times$ Anterior/Posterior & 6.111 & 3.06 & 0.033 & 3.16 & 0023 \\
\hline Exlraversion $\times$ Target/Non-target & 2.37 & 0.94 & -- & 5.12 & 0.011 \\
\hline Extraversion $\times$ Ant $/$ Post $\times$ Target & 6,111 & 7.03 & $<0.001$ & 4.13 & 0.007 \\
\hline
\end{tabular}

Only rows showing significant effects have been included. $P(\mathrm{G}-\mathrm{G})$ indicates probability after Greenhouse-Geisser corretion.

they show a characteristic frontal-maximum topography.) Furthermore, a principal components analysis, not reported here, demonstrated the independence of activity in the ERP channels from EOG activity. Still, to make sure that there were no systematic differences in blink rate, mean amplitudes in the relevant $300-700 \mathrm{msec}$ time band were analyzed in a Task $\times$ Target/Non-target $\times$ Extraversion ANOVA. In this, the only significant effect was that of Target/Non-target. ( $S$ s tended to blink more in this time band when no response had to be delivered.) Specifically, all $F$ s involving Extraversion were $<1$ and non-significant.

A different question concerns testing order. As mentioned in the Introduction, there has been an emphasis on time-on-task in earlier studies, due to the differing influence this has had on personality groups in monotonous tasks. Thus, it was of some importance to determine whether time-on-task had any influence in this study, where tasks were meant to be less monotonous than in the typical oddball paradigm. However, the different tasks were distributed over the testing session by the counterbalancing procedure, and the different influences of task and testing order had to be disentangled to make an assessment of the importance of testing order possible. Therefore amplitudes (peak amplitudes at $\mathrm{Pz}$, as above) were $z$-transformed within each task, and the resulting scores, where the effect of task was removed, were tested for the influence of test block (1.2 or 3 ) and Extraversion. There was no effect of test block, either alone or in interaction. Average z-seore for the first block was 0.006 (SD 1.00), for the second $-0.095 \pm 0.87$ and for the third $0.089 \pm 1.09$. Thus, amplitudes did not diminish over the relatively lengthy testing session, either in the group as a whole, or in extraversion-defined subgroups.

Further, it was mentioned above that Extraversion groups differed in age, albeit within a narrow total range. To separate the possible effects of age from those of Extraversion, age was entered as a covariate in the analyses of peak amplitudes and time-interval mean amplitudes. Age did not exert a significant influence on any effect of interest in those analyses, and the effects involving Extraversion remained significant, except the Extraversion $\times$ Anterior/Posterior interaction for the 300-500 time band. [The main effect of Extraversion on Pz peak amplitude was reduced to borderline significance $(P=0.051)$.] Sex was similarly added to the above analyses, without eliminating any effect involving Extraversion.

Earlier components. As can be seen in Figs 1 and 2, there are at least two clearly delineated components earlier than the P300. They are the P2, around $150 \mathrm{msec}$, and the $\mathrm{N} 2$, around $2(0) \mathrm{m}$ sec. Because these earlier components may also be indicative of cognitive processing, especially the degree of attention paid to the stimuli, they were analyzed with respect to effects of Extraversion. The N2 is a modality-dependent component, and. for visual stimuli, largest over occipital areas. Peak amplitudes from the $\mathrm{O} 1$ and $\mathrm{O} 2$ electrodes were therefore measured and entered into four-way ANOVAs (Extraversion $\times$ Task $\times$ Target/Non-target $\times$ Hemisphere). For the P2, there was a main effect of Extraversion $[F(2,37)=5.03 ; P=0.012]$. Post-hoc tests revealed that the low Extraversion group had significantly lower amplitude (3.69) than the other two groups, which did not differ reliably from each other $(8.00$ vs 6.55$)$. Similarly, there was a main effect of Extraversion on N2 amplitude $[F(2,37)=4.51 ; P=0.018]$, in this case indicating that amplitudes in the Low group were higher (3.94) than in the Medium and High groups (0.24 and 0.75$)$. In both cases. ERP waveforms were more negative-going in the introverted group than in the other two. An analysis of mein amplitude in the 150-300 msec interval confirmed this for the 8 electrodes used in the earlier time-band analyses. There was a main effect of Extraversion $[F(2,37)=4.47 ; P=0.018]$, as well als interactions with the 
anterior-posterior gradient $[F(6,111)=5.01 ; P<0.001]$, and with Target/Non-target $\times$ Anterior/ Posterior $[F(6,111)=2.74 ; P=0.040]$, indicating, as expected, stronger differentiation between personality groups over posterior areas. As seen in Fig. 2, introverts had overall more negative-going traces, starting with the time interval including the $\mathrm{P} 2$ and $\mathrm{N} 2$, and continuing throughout the duration of the late positive complex.

It could be asked whether the difference between groups was a reflection of pre-stimulus differences rather than post-stimulus processing. In conformity with standard procedures, a pre-stimulus baseline was computed and subtracted from the post-event ERP in this study. However, if there were systematic differences in pre-stimulus level, such as could be caused by CNV-related slow potentials built on expectancy of the upcoming stimulus, subtraction of this baseline level could introduce a bias in the post-stimulus ERP. Therefore, the pre-stimulus level was examined for influence of Extraversion, Task and Target/Non-target in 3-way ANOVAs for the $\mathrm{Cz}$ and $\mathrm{Pz}$ electrodes. In these analyses, no effect was significant, and it can therefore safely be concluded that no bias was introduced by the baseline correction procedure.

Impulsivity or sociability? To examine whether the found effects of Extraversion can be ascribed to one of its subcomponents, Impulsivity and Sociability. more than the other, a separate analysis was undertaken. In this, an overall measure of P300 amplitude was computed as the average, across tasks, of $\mathrm{Pz}$ peak amplitude for target stimuli. This variable correlated more highly $(r=0.39)$ with Impulsivity than with Sociability $(r=0.24)$, or with the composite Extraversion score $(r=0.36)$.

A stepwise regression analysis was performed, in which Impulsivity, Sociability, Neuroticism, Age and Sex were offered to the analysis program as predictors of the P300 amplitude. Only Impulsivity was entered into the model, all the other variables giving $F$-values below the set $F$-to-enter criterion of 4.0. This one-predictor model gave a significant regression effect, $F(1,38)=6.65$; $P=0.014$. Hence, the personality effect of P300 amplitude can be ascribed primarily to the impulsivity component of extraversion, independently of age, sex and other examined personality aspects.

\section{I S C USS ION}

The main finding of this study was the clear difference in P300 amplitude between Extraversion groups. For all measures studied, amplitude increased with increasing degrees of extraversion. Although this linear ordering of amplitudes across the Low, Medium and High Extraversion groups was evident for the late positive complex evoked by both target and non-target stimuli, it was largest for target stimuli in posterior derivations. The topography and the target effect are those of the classical P300.

In morphology and topography, the endogenous ERP components showed distinct effects of the visual classification tasks used in this study. There was a right-over-left asymmetry of amplitudes, in keeping with the well-known right-hemispheric advantage for visuospatial tasks. All tasks evoked large late positivities in the $300-700 \mathrm{msec}$ range. Classification based on simple visual features (black/white) gave higher amplitudes for, and a shorter duration of, the late positivity than the semantically based classification. The conjunction task yielded, as could be expected, the most extended duration, and also the lowest amplitude, possibly a function of greater latency variability in single trials. Interestingly, the waveshape in the conjunction task combined features from both the simpler ones (cf. the occipital traces for target stimuli in Fig. 1). The earlier P150-N200 complex resembled that of the colour selection task, and the late positivity resembled that of the semantic task, although with a biphasic peak. corresponding to the latencies for the two constituent tasks. The waveshapes suggest a sequential selection process, based on extraction of the more accessible simple features first.

The late positive complex, which is of prime interest for the extraversion effects, may have merged elements of the Slow Wave (Sutton \& Ruchkin, 1984) with those of the classical P3b. The topography and the target effect are those characteristic of the P3b, but the extended duration suggest that the Slow Wave may also be involved. A broad separation of these effects has been made here by the separate analyses of the 300-500 and 500-700 msec time bands. Under other circumstances, a more sensitive disentangling of overlapping components can be accomplished by Principal Components Analysis, 
but it pre-supposes constant latency of components over tasks, which is not the case here. For the assessment of extraversion-related differences, the distinction between $\mathrm{P} 3 \mathrm{~b}$ and Slow Wave is of minor importance, since the extraversion effects were similar over the two time bands.

The earlier 150-300 msec time band, encompassing the N200, also showed a separation between personality groups, with the introverted group showing the largest N200. Negative-going activity in this time range usually reflects attentive processing of the evoking stimulus, with this processing negativity (Näätänen, Gaillard \& Montysalo, 1978) often being superimposed on other components in the time band. Thus, it can at least be concluded that failing attention in the introverted group is not the explanation for the fact that extraverts had higher P300 amplitudes.

A number of alternatives, including recording and analysis artifacts, were considered and rejected as possible mediators in the observed relation between extraversion and ERP amplitude. The influence of EOG artifacts, which is minimal at posterior recording sites in any case, was found to have been successfully removed by the correction procedure. In addition, the size of the EOG deflections showed no systematic variation between personality groups. Variations in pre-stimulus baseline did not account for the observed ERP amplitude differences. Demographic variables, such as age and sex, did not mediate the relationship between extraversion and amplitude.

How, then, are the present findings to be interpreted? The most immediate observation to make regarding the present results is that they are at variance with much of the extant literature. Although neither voluminous nor entirely concordant, a corpus of findings support the view that introverts show larger P300s than extraverts (Daruna et al., 1985; Ditraglia \& Polich, 1991; Wilson \& Languis, 1990). However, these studies have all adhered to some variant of the auditory oddball paradigm, which, as described in the Introduction, tends to induce habituation and a gradual decay of attentional resources devoted to the task as the experiment progresses. Indeed, this vigilance-like nature of the task has been part of the rationale for expecting differences between extraverts and introverts to emerge. As discussed by the authors (Daruna et al., 1985; Ditraglia \& Polich, 1991), attentional resources devoted to the task seem to decrease at a more rapid pace in extraverts than in introverts under monotonous conditions. However, in the early part of a recording session (Ditraglia \& Polich, 1991), or under conditions where habituation is minimized (Cahill \& Polich, 1992; Orlebeke et al., 1989). extraverts tend to produce larger P300s.

The present study attempted to unconfound the issues of attention decay and P300 differences. The rate of events was kept high: the stimuli were more varied, and more processing was required of the Ss than in the typical auditory oddball task. The results indicate that the manipulations were successful in this respect. There was no decline in amplitudes over the experimental session. Performance was high, and equal between Extraversion groups. The absence of group differences was not due to a general ceiling effect or to lack of sensitivity in the performance measures, as shown by the significant performance difference between Neuroticism groups.

It is worth observing that a dissociation between performance measures and the P300 is not an uncommon finding. Here, as in many other studies, reaction time was notably shorter than P300 latency. In other words, when the P300 is at its peak, the response has already been launched and delivered. It would therefore be a mistake to expect a simple relation between performance and P300 amplitude. except in the limiting case where the $S$ lapses in his/her attention and fails to develop both P300 and the appropriate behavioural response. In more general terms, the P300 is a consequence of stimulus evaluation, not a part of it.

What reasons can there be for expecting interindividual differences in P300 amplitude, given that factors such as attention and motivation are equalized between groups? Models of P300 amplitude have normally concentrated on the task and stimulus factors that explain within- $S$ variance (Johnson, 1986). Between-Ss differences have not been the topic of extensive systematizing efforts, and theoretical models can therefore only tentatively be applied to the question of individual differences. As described in the Introduction, biophysical considerations vindicate the view that slow cortical positivities are a reflection of temporarily reduced excitability due to hyperpolarization of apical dendrites (Birbaumer et al., 1990). Correspondingly, slow cortical negatives reflect depolarization and lowered thresholds for excitation. An experiment with a secondary reaction time (RT) task has shown that probe RTs were slowed in the time range where P30)0s were evoked (Woodward, Brown, Marsh \& Dawson, 1991), in consistency with the authors view that the P300 manifests a brief, widely distributed, inhibitory event. 
The importance of the putatively inhibitory nature of the P300 for the question of individual differences comes from the fact that several views of the biological basis of extraversion have assumed that extraverts are more prone to develop inhibition than introverts. The earlier version of Eysenck's theory (1957) was couched in the language of inhibition and excitation. and so was the later development of Brebner and Cooper (1974; Brebner, 1990). Although the empirical findings on which the inhibition theory of extraversion was based would probably be described in other terms today (Eysenck \& Eysenck, 1985), the possibility remains that transient states of inhibition following perceptual processing may be a characteristic contributing to individual differences in extraversion. The findings from memory experiments, where extraverts show better retrieval at short retention intervals, and introverts better at long, have been interpeted to mean that more sustained post-processing, interfering with short-term retrieval but facilitating long-term retention, takes place in introverts (Howarth \& Eysenck, 1968). A more distinct phase of perceptual closure in extraverts, accompanied by transient inhibition, is compatible with earlier findings of extraverts having lower critical flicker fusion thresholds (Simonson \& Brozek, 1952), shorter duration of aftereffects (Levy \& Lang, 1966), and more involuntary rest pauses in a tapping task (Eysenck, 1964). In a neurophysiological context, extraverts have been found to display reduced motoneural excitability as assessed by reflex recovery functions and homosynaptic depression procedures (Pivik, Stelmack \& Bylsma, 1988). In other words, the recovery to full discharge capability after afferent impulses took longer in extraverts than in introverts.

The hypothesis that extraverts differ from introverts in transient inhibition is open to empirical test with secondary RT methodology of the kind used by Woodward et al. (1991). If the conjecture is right, the difference in inhibitory potential (extraverts $>$ introverts) is masked in monotonous vigilance tasks by the growing inattention of extraverts during the course of the task. Only if stimuli are attended to can they elicit the inhibition signalled by the P300. Since the inhibition accompanies the last phases of perceptual analysis, or follows the termination of it, it need not affect performance, although it does affect P300 amplitude.

Finally, it was found that the differences in P300 amplitude can be ascribed to the impulsivity subtrait of extraversion rather than the sociability component. In this respect, the findings concur with several studies linking biologically based differences between extraverts and introverts primarily to the impulsivity component (Eysenck \& Levey, 1972; O'Gorman \& Lloyd, 1987: Revelle et al., 1980; Stenberg, 1992).

Acknowledgements-This research was supported by grants from the Bank of Sweden Tercentenary Foundation, the Swedish Research Council for the Humanities and Social Sciences and the Swedish Medical Research Council. I am grateful to Professor Ingmar Rosen for valuable comments and to Mrs Christina Lindbom for skilled technical assistance.

\section{REFERENCES}

Birbaumer, N. \& Elbert, T. (1988). P3: Byproduct of a byproduct. Behavioral and Brain Sciences, 11, 375-376.

Birbaumer, N., Elbert, T., Canavan, A. G. M. \& Rockstroh, B. (1990). Slow potentials of the cerebral cortex and behaviour. Phisiological Reviews, 70, 1-41.

Brebner, J. (1990). Psychological and neurophysiological factors in stimulus-response compatibility. In Proctor, R. W. \& Reeve, T. G. (Ed.), Stimulus-response compatibility (pp. 24l-260). Amsterdam: Elsevier.

Brebner, J. \& Cooper, C. (1974). The effect of a low rate of regular signals upon the reaction time of introverts and ex traverts. Journal of Research in Personality, 8, 263-276.

Bruneau, N.. Roux, S.. Perse, J. \& Lelord, G. (1984). Frontal evoked responses, stimulus intensity control, and the extraversion dimension. Annals of the New York Academy of Sciences, 425, 546-550.

Cahill, J. M. \& Polich, J. (1992). P300, probability, and introverted/extraverted personality types. Biological Psychology, 33. $23-35$.

Connolly, J. F. \& Kleinman, K. M. (1978). A single channel method for recording vertical and lateral eye movements. Electroencephalography and Clinical Neurophysiology, 45, 128-129.

Costa, P. T. \& McCrae, R. R. (1992). Four ways five factors are basic. Personality and Individual Differences, 13, 653-665.

Daruna. J. H., Karrer, R. \& Rosen, A. J. (1985). Introversion, attention and the late positive component of event-related potentials. Biological Psychologi, 20, 249-259.

Deecke. L. \& Lang, W. (1988). P300 as the resolution of negative cortical DC shifts. Behavioral and Brain Sciences, 1/, 379-381.

Ditraglia, G. M. \& Polich, J. (1991). P300 and introverted/extraverted personality types. Psychophysiology, 28, $177-184$.

Donchin, E. \& Coles, M. G. H. (1988), Is the P300 component a manifestation of context updating? Behavioral and Brain Sciences, $1 /, 357-374$.

Eysenck, H. J. (1957). The dynamics of anxiety and hysteria. London: Routledge and Kegan Paul. 
Eysenck. H. J. (1964). Involuntary rest pauses in tapping as a function of drive and personality. Perceptual and Motor Skills, $18,173-174$

Eysenck, H. J. (1967). The biological basis of personality. Springfield, IL: Thomas

Eysenck, H. J. (1992). Four ways five factors are not basic. Personality and Individual Differences, 13, 667-673.

Eysenck, H. J. \& Eysenck, M. W. (1985). Personality and individual differencer. London: Plenum.

Eysenck, H. J. \& Levey, A. (1972). Conditioning, introversion-extraversion and the strength of the nervous system. In Nebylitsyn, V. D. \& Gray, J. A. (Ed.), Biological hases of individual behavior. New York: Academic Press.

Friedman, J. \& Meares, R. (1979). Cortical evoked potentials and extraversion. Psychosomatic Medicine, 4l, $279-286$.

Fulker, D. W. (1981). The genetic and environmental architecture of psychoticism, extraversion and neuroticism. In Eysenck, H. J. (Ed.). A model for personality (pp. 88-123). Berlin: Springer.

Gale, A. (1983). Electroencephalographic studies of extraversion-introversion: a case study in the psychophysiology of individual differences. Personality and Individual Differences. 4, 371-380.

Gray, J. A. (1972). The psychophysiological nature of introwersion-extraversion: a modification of Eysenck's theory. In Nebylitsyn, V. D. \& Gray, J. A. (Ed.), Biological bases of individual behavior (pp. 182-205). London: Academic Press.

Gray, J. A. (1981). A critique of Eysenck's theory of personality, In Eysenck. H. J. (Ed.), A model for personality (pp. 246-276). Berlin: Springer.

Howarth. E. \& Eysenck, H. J. (1968). Extraversion, arousal, and paired associate recall. Journal of Experimental Research in Personalin, 3,114-116.

Johnson, R. (1986). A triarchic model of P300 amplitude. Psychophysiology, 23, 367-384.

Keister, M. E. \& McLaughlin, R. J. (1972). Vigilance performance related to extraversion-introversion and caffeine. Journal of Experimental Research in Personality, 6, 5-11.

Lemere, F. (1936). The significance of individual differences in the Berger rhythm. Brain, 59, 366-375

Levy, P. \& Lang, P. J. (1966). Activation, control and the spiral aftermovement. Journal of Personality and Social Psychology, $3,105-112$.

Mathew, R. J., Weinman, M. L. \& Barr, D. L. (1984). Personality and regional cerebral blood flow. British Journal of Psychiatr, 144, 529-532.

Näätänen. R.. Gaillard, A \& Montysalo, S. (1978). The Nl effect of selective attention reinterpreted. Acta Psvchologica, 42, $313-329$.

O'Connor, K. (1983). Individual differences in components of slow cortical potentials: implications for models of information processing. Personality and Individual Differences, 4, 40.3-410.

O'Gorman. J. (1977). Individual differences in habituation of human physiological responses: A review of theory, method, and findings in the study of personality correlates in non-clinical populations. Biological Psychology. 5 , $257-318$

O'Gorman. J. G. (1984). Extraversion and the EEG: I. An evaluation of Gale's hypothesis. Biological Psychology, 19,95-1 12

O'Gorman, J. G. \& Lloyd, J. E. (1987). Extraversion, impulsiveness, and EEG alpha activity. Personality and Indivilual Differences, $8,169-174$.

Orlebeke, J. F., Kok, A. \& Zeillemaker, C. W. (1989). Disinhibition and the processing of auditory stimulus intensity: an ERP study. Personality and Individual Differences, 10, 445-451.

Pfefferbaum, A., Ford, J. M., Wenegrat, B. G., Roth, W. T. \& Kopell, B. S. (1984). Clinical application of the P3 component of event-related potentials. I. Normal aging. Electroencephalography and Clinical Neurophysiology, 59, 85-103.

Picton, T. W. \& Hillyard, S. A. (1988). Endogenous event-related potentials. In Picton, T. (Ed.), Human event-related potentials. Amsterdam: Elsevier.

Pivik, R. T., Stelmack, R. M. \& Bylsma, F. W. (1988). Personality and individual differences in spinal motoneural excitability Psvchophssiologv, 25, 16-24.

Polich. J. \& Martin. S. (1992). P300, cognitive, and personality: a correlational study of university undergraduates. Personality and Individual Differences, 13,533-543.

Pritchard, W. S. (1989). P300 and EPQ/STPI personality traits. Personality and Individual Differences, 10, 15-24.

Revelle, W., Humphreys, M. S., Simon, L. \& Gilliland, K. (1980). The interactive effect of personality, time of day, and caffeine: a test of the arousal model. Journal of Experimental Psychology: General, 109, 1-31.

Siddle, D. A., Morrish, R. B., White. K. D. \& Mangan, G. L. (1969), Relation of visual sensitivity to extraversion. Journal of Experimental Research in Personality, 3, 264-267.

Simonson, E. \& Brozek, J. (1952). Flicker fusion frequency. Physiological Review, 32. 349-378.

Snodgrass, J. G. \& Vanderwart. M. (1980). A standardized set of 260 pictures: Norms for name agreement, image agreement, familiarity, and visual complexity. Joumal of Experimental Psychology: Human Learning and Memory, 6, $174-215$.

Soskis, D. A. \& Shagass, C. (1974). Evoked potential tests of augmenting-reducing. Psychophysiology, $/ I, 175-190$.

Stelmack, R. M. \& Campbell, K. B. (1974). Extraversion and auditory sensitivity to high and low frequency. Perceptual and Motor Skills, 38, 875-879.

Stelmack, R. M. \& Michaud. A. A. (1985). Extraversion, attention, and habituation of the auditory evoked response. Journal of Research in Personality, 19, 416-428.

Stelmack, R. M., Achorn, E. \& Michaud. A. (1977). Extraversion and individual differences in auditory evoked response. Psychophysiology, 14, 368-374.

Stenberg, G. (1992). Personality and the EEG: arousal and emotional arousability. Personality and lndividual Differences, 13, 1097-1113.

Stenberg, G., Rosén, I. \& Risberg. J. (1988). Personality and augmenting/reducing in visual and auditory evoked potentials. Personality and Individual Differences, 9. 571-579.

Stenberg, G. Rosén. I. \& Risberg, J. (1990a). Attention and personality in augmenting/reducing of visual evoked potentials. Personality and Individual Differences, $11,1243-1254$

Stenberg, G., Wendt, P. E. \& Risberg, J. (1993). Regional cerebral blood fow and extraversion. Personality and Indiridual Differences, 15, 547-554.

Stenberg, G., Risberg, J., Warkentin, S. \& Rosén, I. (1990b). Regional patterns of cortical blood flow distinguish extraverts from introverts. Personality and Individual Differences, II, 66.3-67.3.

Sutton, S. \& Ruchkin, D. (1984). The late positive complex: Advances and new problems. In Karrer. R., Cohen. J. \& Tueting, P. (Ed.), Brain and information (pp. 1-23). New York: Plenum. 
Vasey, M. W. \& Thayer, J. F. (1987). The continuing problem of false positives in repeated measures ANOVA in psychophysiology: a multivariate solution. Psychophysiology, 24, 479-486.

Verleger, R. (1988). Event-related potentials and cognition: A critique of the context updating hypothesis and an alternative interpretation of the P3. Behavioral and Brain Sciences, 11, 343-356.

Wilson, G. D. (1981). Personality and social behaviour. In Eysenck, H. J. (Ed.). A model for personality. Berlin: Springer.

Wilson, M. A. \& Languis, M. L. (1990). A topographic study of differences in the P300 between introverts and exIraverts. Brain Topography, 2, 269-274

Woodward, S. H., Brown, W. S., Marsh, J. T. \& Dawson, M. E. (1991). Probing the time course of the auditory oddball P3 with secondary reaction time. Psychophysiology, 28,609-618.

Zuckerman, M. (1991). Psychobiology of personaliny. Cambridge: Cambridge University Press.

Zuckerman, M. (1992). What is a basic factor and what factors are basic? Turtles all the way down. Personality and Individual Differences, 13, 675-681. 